\title{
Fibronectin fragments in human seminal plasma
}

\author{
Iwona Kątnik-Prastowska ${ }^{1}$, Magdalena Przybysz ${ }^{1}$ and Anna Chełmońska-Soyta ${ }^{2}$ \\ ${ }^{1}$ Department of Chemistry and Immunochemistry, Medical University, Wroctaw, Poland; ${ }^{2}$ Institute of Immunol- \\ ogy and Experimental Therapy, Polish Academy of Sciences, Wroctaw, Poland; \\ 凶e-mail: iwona@immchem.am.wroc.pl
}

Received: 13 May, 2004; revised: 22 November, 2004 accepted: 10 December, 2004

available on-line: 15 May, 2005

\begin{abstract}
The study has revealed the presence of fibronectin (FN) fragments and a lack of intact FN in 72 seminal plasma samples. The FN fragmentation was examined by immunoblotting with a monoclonal antibody specific to the central cellular FN domain and was confirmed with a monoclonal antibody directed to the C-terminal domain of FN. Nine FN fragments between 60 and $200 \mathrm{kDa}$ and five fragments of 60-150 kDa were identified in seminal plasma samples of normozoospermic and of terato-, oligoterato-, and oligoasthenoterato-spermic groups, respectively. The relative amounts of the 60,90 and $100 \mathrm{kDa}$ FN fragments were 2-3 times higher in seminal plasmas with abnormal semen characteristics than in the normozoospermic group. The results suggest that seminal plasma FN fragments may contribute to fertilization and the analysis of FN fragmentation may have a diagnostic value in andrological investigations.
\end{abstract}

Keywords: fibronectin, seminal plasma, cellular domain of fibronectin

Fibronectin (FN) is a ubiquitous multifunctional adhesive glycoprotein, widely distributed in human and animal tissues. It is known to participate in various cellular regulation processes, including cell growth and migration, tissue remodeling and differentiation, hemostasis, as well as wound healing. This is accomplished by FN binding domains specific for cells, DNA, collagen, heparin, and fibrin (reviewed by Romberger, 1997, and by Labat-Robert, 2002). On various types of cells, including spermatozoa, FN exists in an oligomeric, insoluble form, whereas in the pericellular matrix it is an insoluble, polymeric, extracellular filament protein. On the other hand, soluble monomeric FN was found in blood and seminal plasmas as well as in other body fluids. FN is largely synthesized in the liver by hepatocytes, but the cellular form is produced locally by many cell types (reviewed by Romberger, 1997).

FN has been found in tissues and fluids of the male reproductive tract. It is a component of spermatozoa and the basement membrane of the testis (Pinke et al., 1997) as well as of epididymal and seminal fluid (Stauffer \& Parsons, 1989; Wennemuth et al., 2000). However, the biological func- tion of FN in semen and on spermatozoa is still undefined. Pinke et al. (1997) have suggested that FN may bind to the cellular components that are exposed when the sperm is damaged and then aid macrophages in ingesting the spermatozoa, providing an opportunity for selecting out abnormal spermatozoa. Wennemuth et al. (2000) have reported on a significant negative correlation between FN concentration and total sperm motility. Clinical studies of Stauffer and Parsons (1989) and Wennemuth et al. (2000) have indicated that determination of FN concentration in seminal plasma can be recommended as an additional diagnostic tool in andrology.

The aim of this study was to identify degradation products of $\mathrm{FN}$ in the seminal plasma and then to determine if the presence of FN fragments recognized by a monoclonal antibody directed to the central cellular domain of FN could be associated with the results of semen evaluation. Western immunoblotting was used to visualize the molecular masses and the range of FN fragmentation. The differences among seminal plasma FN fragments were qualitative and quantitative and were related to the results of semen analysis and to sperm morphology. 


\section{MATERIALS AND METHODS}

Sources of seminal and blood plasmas. Seminal ejaculates (69) were collected from male partners (20-41 years old) of infertile couples, directed by andrologists to Wrocław Semen Laboratory for semen analysis. Additionally, three samples from healthy donors (33-45 years old) with proved fertility were included into the studies. The ejaculates were collected by masturbation into sterilized tubes after a minimum 3 days of sexual abstinence. The ejaculates were allowed to stand at $37^{\circ} \mathrm{C}$ no longer than $1 \mathrm{~h}$ until the liquefaction was completed and standard sperm analysis (volume, $\mathrm{pH}$, morphology, concentration, motility, viability) was carried out according to the recommendations of the World Health Organization. The seminal samples were centrifuged at $3500 \times g$ for $10 \mathrm{~min}$ at room temperature to obtain plasma. The seminal plasma divided into small aliquots with and without protease inhibitor $(1 \mathrm{mM}$ phenylmethylsulfonyl fluoride) was frozen at $-76^{\circ} \mathrm{C}$ until used. The seminal plasma FN did not show any additional degradation during 3 months of storage (not shown).

On the basis of the overall semen analyses the following seminal plasma groups were distinguished: 1) Normozoospermic ( $N, n=19)$, when the amount of spermatozoa was not less than $2 \times 10^{7}$ per $\mathrm{ml}$, and of which more than $30 \%$ expressed correct sperm morphology with a motility of $50 \%$ or more $1 \mathrm{~h}$ after ejaculation. This group was divided into subgroups $\mathrm{N}_{1}$ and $\mathrm{N}_{2}$, which consisted of the three samples derived from men with proved fertility and 16 samples from men suspected of infertility, respectively; 2$)$ Teratozoospermic: $(T, n=26)$, with less than $30 \%$ of morphologically normal forms; 3 ) Oligoteratozoospermic: (OT, $\mathrm{n}=10)$, with mixed anomalies: less than $30 \%$ of morphologically normal forms and sperm concentration $<2 \times 10^{7} / \mathrm{ml} ; 4$ ) Oligoasthenoteratozoospermic: (OAT, $\mathrm{n}=17$ ), with mixed anomalies: sperm motility $<50 \%$ or progressive motility $<25 \%$; less than $30 \%$ of morphologically normal forms and sperm concentration $<2 \times 10^{7}$ $/ \mathrm{ml}$. Blood plasma samples were taken from healthy both sexes volunteers (28-45 years old) working in the Medical University.

FN concentration. The concentration of plasma FN was determined by standard sandwich type solid phase enzyme linked immunoassays (ELISA) using as a catching agent an anti-FN monoclonal antibody with specificity to the cellular domain of FN (Sigma Chemical Co., St Louis, MO, USA, FN15; 100 $\mu \mathrm{l}$ of a 1:10000 dilution per well). Human plasma FN (Sigma, 10 to $50 \mathrm{ng} /$ well) was used as a standard. The amount of plasma FN bound by the coating monoclonal antibody was quantified by reaction with rabbit polyclonal anti-FN antibodies (Sigma, diluted 1:10000). Peroxidase-conjugated goat anti- rabbit immunoglobulins (Sigma, diluted 1:10000) were the secondary antibodies. The amount of FN was assayed by a colorimetric reaction using o-phenylenediamine dihydrochloride $/ \mathrm{H}_{2} \mathrm{O}_{2}$ as the enzyme substrate and measured in Stat Fax 2100 Reader at $450 \mathrm{~nm}$ and $630 \mathrm{~nm}$ as a reference filter. All ELISA immunobinding and washing steps were carried out in TBS containing $0.1 \%$ Tween 20, pH 7.3.

Western immunoblot analysis. Seminal plasma samples diluted with $0.06 \mathrm{M}$ Tris $/ \mathrm{HCl}, \mathrm{pH} 6.8$ containing $3 \%$ SDS (w/v), 10\% glycerol (v/v) and 5\% 2-mercaptoethanol $(\mathrm{v} / \mathrm{v})$ were incubated before electrophoresis at room temperature for $1 \mathrm{~h}$. Then, 300 ng of FN, determined by ELISA, was subjected to SDS-polyacrylamide gel electrophoresis on a $5-16 \%$ gradient gel according to Laemmli (1970) in a Sigma vertical electrophoresis cell. After electrophoresis, protein separation was controlled by staining with $0.2 \%$ Ponceau $\mathrm{S}$ in acetic acid. The separated proteins were subsequently blotted onto nitrocellulose (Serva Electrophoesis GmbH, Heidelberg, Germany) using standard protocols (transfer buffer: $0.025 \mathrm{M}$ Tris, $0.19 \mathrm{M}$ glycine, $20 \%$ methanol (v/v), $\mathrm{pH}$ 8.3; $100 \mathrm{~V}, 150 \mathrm{~mA}$ for $1 \mathrm{~h}$ ). After transfer, the nitrocellulose membrane was incubated with $5 \%$ casein in TBS (Tris-buffered saline, $\mathrm{pH} 7.5$ ) overnight at $4^{\circ} \mathrm{C}$ to block non specific protein-binding sites. Then, the blots were incubated at room temperature for 1 $\mathrm{h}$ with mouse monoclonal antibody anti-human FN recognizing the cellular domain of FN (FN-15 Sigma as well as FN 30-8, code M010, TaKaRa), diluted $1: 10000$ in $5 \%$ casein in TBS. Independently, as a control, some of the nitrocellulose blots were processed with an anti-human plasma fibronectin carboxy-terminal domain monoclonal antibody, clone

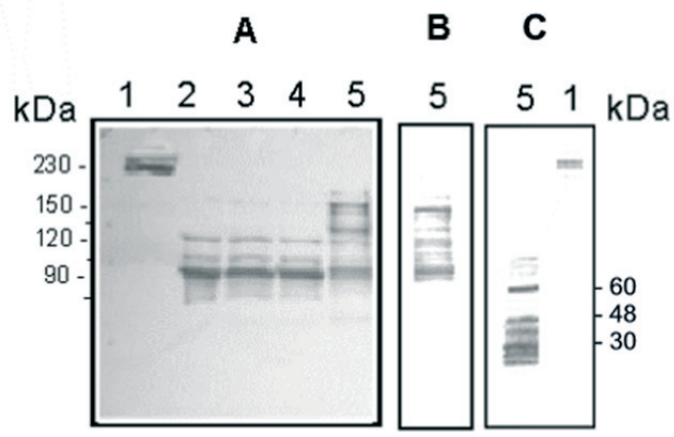

Figure 1. Fibronectin fragment patterns in seminal plasma revealed by immunoblotting.

SDS/PAGE was done according to Laemmli (1970) under reducing conditions in $\mathbf{A}$ and $\mathbf{C}$, and under non-reducing in B. The immunoblots were developed with monoclonal antibodies directed to cellular domain of $\mathrm{FN}(\mathbf{A}, \mathbf{B})$ or with ones directed to carboxy-terminal sequences of FN (C). In all cases $300 \mathrm{ng}$ of FN was applied per lane. Lane 1 represents the pattern for blood plasma $\mathrm{FN}$ and lanes 2-5 for seminal plasmas with the following semen characteristics: 2-oligoasthenoteratozoospermic; 3-oligoteratozoospermic; 4-teratozoospermic; 5-normozoospermic. 
868A11 (MAB 1935, Chemicon), diluted 1:10000. After extensive washing, the blots were incubated with rabbit anti-mouse immunoglobulins conjugated to horseradish peroxidase (Sigma, 1:10000 dilution in 5\% casein in TBS). The colour reaction was developed with diaminobenzidine. Then, the blots were dried and analyzed. Bands corresponding to particular proteins were scanned and then analyzed by densitometry using SigmaGel (Gel Analysis Software version 1.0, Jandel Scientific Software, San Rafael, Canada).

Assessment of FN fragments. The apparent molecular mass of FN bands in SDS-electrophoresis were determined by comparisson with BioRad molecular mass protein standards and intact human FN (230 kDa; Sigma). The relative amount of particular FN fragments was expressed as the percentage of the total number of pixels, in a lane. For this purpose, the blots were scanned and analysed by densitometry using SigmaGel Spots Mode Measurements (Jandel Scientific Software).

\section{RESULTS AND DISCUSSION}

Western immunoblot analysis of seminal plasma samples under reducing (Fig. 1A: lanes 2-5) or non-reducing (Fig. 1B, lane 5) conditions with the use of a monoclonal antibody directed to the central, cellular domain of FN (Fig. 1A) revealed a lack of the intact FN molecule. The fragmentation of seminal plasma FN was confirmed by the use of a monoclonal antibody to the C-terminal domain of FN (Fig. 1C, lane 5). In contrast, under the same conditions of the analysis, blood plasma FN did not show any fragmentation (Fig. 1A, C, lane 1) and two bands corresponding to the FN polypeptide chains forming the native molecule were visible.

The differences among the seminal plasma FN fragment patterns revealed by the monoclonal antibody directed to the cellular domain of FN were qualitative and quantitative and they were related to the results of semen analysis, mainly to sperm morphology. The relative amounts of particular FN fragment bands are given in Table 1. Nine FN fragments of $60-200 \mathrm{kDa}$ were identified in normozoospermic seminal plasma samples derived from fertile men as well as from those suspected infertility. In teratozoospermic, oligoteratozoospermic, oligoasthenoteratozoospermic seminal plasma samples the monoclonal antibody elicited only five bands of 60-120 kDa (Fig. 1, Table 1). All seminal plasma samples contained the 60, 90, 100 and $120 \mathrm{kDa} F N$ fragments. The bands of 95, 125, 150, 160 and 200 $\mathrm{kDa}$ were present in normozoospermic seminal plasmas, but were undetectable in the samples with abnormal semen parameters. In contrast, the $75 \mathrm{kDa}$ fragment was present in pathological seminal plasmas, but was absent in the normozoospermic ones. The relative amounts of the 60,90 and $100 \mathrm{kDa} F N$ fragments were 2-3 times higher in the pathological samples than in the normozoospermic group.

The appearance of FN fragments has been mainly reported in pathological biological fluids: in cancer (Labat-Robert, 2002), arthritis (Barilla \& Carsons, 2000), periodontal disease (Huynh et al., 2002) and have also been detected in seminal plasma by the use of polyclonal antibodies (Lilja et al., 1987).

The FN fragments could have several functions not shared by the intact molecule, suggesting that proteolysis leads to the uncovering of cryptic activities and/or a loss of FN functions they had

Table 1. Relative amounts of FN fragments in seminal plasma

\begin{tabular}{|c|c|c|c|c|c|c|}
\hline \multirow[t]{2}{*}{$\begin{array}{l}\text { FN bands } \\
(\mathrm{kDa})\end{array}$} & \multicolumn{5}{|c|}{$\begin{array}{l}\text { FN fragments in seminal plasma } \\
\qquad(\%)\end{array}$} & \multirow{2}{*}{$\begin{array}{c}\text { FN bands } \\
\text { in blood plasma }(\mathrm{kDa}) \\
\mathrm{n}=4\end{array}$} \\
\hline & $\begin{array}{r}\mathbf{N}_{1} \\
\mathrm{n}=3\end{array}$ & $\begin{array}{r}\mathbf{N}_{2} \\
\mathrm{n}=16\end{array}$ & $\begin{array}{r}\mathrm{T} \\
\mathrm{n}=26\end{array}$ & $\begin{array}{r}\text { OT } \\
\mathrm{n}=10\end{array}$ & $\begin{array}{r}\text { OAT } \\
\mathrm{n}=17\end{array}$ & \\
\hline 240 & & & & & & 45 \\
\hline 230 & & & & & & 55 \\
\hline 200 & $9.0 \pm 4$ & $10.0 \pm 6$ & & & & \\
\hline 160 & $15.3 \pm 12$ & $16.6 \pm 7$ & & & & \\
\hline 150 & $17.3 \pm 6$ & $25.3 \pm 4$ & & & & \\
\hline 125 & $9.3 \pm 2$ & $10.1 \pm 6$ & & & & \\
\hline 120 & $11.4 \pm 2$ & $8.4 \pm 4$ & 16.6 & 11.1 & 13.3 & \\
\hline 100 & $4.8 \pm 8$ & $1.6 \pm 4$ & 16.9 & 13.3 & 9.3 & \\
\hline 95 & $11.5 \pm 4$ & $5.6 \pm 4$ & & & & \\
\hline 90 & $20.0 \pm 14$ & $21.3 \pm 8$ & 43.0 & 50.7 & 49.8 & \\
\hline 75 & & & 14.6 & 16.0 & 17.8 & \\
\hline 60 & $1.2 \pm 2$ & $1.1 \pm 0.5$ & 8.9 & 8.8 & 9.7 & \\
\hline
\end{tabular}

The FN fragments were revealed by SDS-electrophoresis and immunoblotting with monoclonal antibody directed to cellular domain of FN as described in Materials and Methods. $\mathrm{N}_{1}$, normozoospermic from men with proved fertility, and $\mathrm{N}_{2}$, normozoospermic from patients suspected of infertility; T, teratozoospermic; OT, oligoteratozoospermic; OAT, oligoasthenoteratozoospermic. For T, OT and OAT groups " $\mathrm{n}$ " indicates the number of seminal plasma samples, equal aliquots of which were pooled. 
before proteolysis (Fukai et al., 1995; Yamada, 2000; Tchougounova et al., 2001). It is not known why FN fragments show activities that differ from those of intact FN. Hashino et al. (1996) have reported that proteolytic fragments of 70-120 kDa derived from the cell binding domain of $\mathrm{FN}$ retains full activity in the adhesion assay, but a smaller fragment of $11.5 \mathrm{kDa}$ has only $1-5 \%$ of the activity. Grant et al. (1998) have shown, that the activity of a band of 90 $\mathrm{kDa}$ represented matrix metalloproteinase-2 (MMP2) tightly associated with the FN fragment, and the association of this fragment inhibited autoactivation of MMP-2. According to Yi \& Ruoslahti (2001), a 76 $\mathrm{kDa}$ FN-derived peptide showed an antiangiogenic and antimetastatic effect in vitro.

The FN in human seminal fluid originates from seminal vesicles and ampullae and is incorporated in the framework of the seminal gel formed during the immediate postejaculatory phase. The gel-bound fibronectin together with a high molecular mass seminal vesicle protein - semenogelin - was found to be progressively fragmented by an abundant prostatic kallikrein-like protease (prostate specific antigen) and then to be released into the seminal fluid during lysis of the gel structure (Lilja et al., 1987). It seems most probable that some FN fragments rather than the intact FN molecule play a significant role in the seminal plasma. The FN fragments can act as competitive inhibitors of sperm FN interaction with integrins present on the surface of genital tract epithelia, or, conversely, the binding of FN peptide could augment integrin signaling (Stanton et al., 1999; Wassarman \& Litscher, 2001) during fertilization. The soluble FN fragments might be involved in modulation of leukocyte function which may protect normal sperm from ingestion during postcoital leukocytosis. Moreover, the seminal plasma can be a source of fibronectin fragments for coating spermatozoa, and thus protects the male germ cells from infection. On the other hand, the seminal plasma FN fragments can act as an active participant in intercellular recognition, or they could play a protective role in the complex route of spermatozoa to the oocyte (James \& Skibiński, 1995). There is a need for further studies, particularly for an assessment of the activity of metalloproteinases 2 and 9, and their inhibitors in relation to the appearance of $\mathrm{FN}$ fragments. Moreover, it seems to be of great interest to isolate the fibronectin fragments from seminal plasma and then to demonstrate their biological activities as well as to elucidate the differences between fragments derived from fertile and infertile men.

\section{REFERENCES}

Barrila ML, Carsons SE (2000) Semin Arthritis Rhem 29: 252-265.

Fukai F, Ohtaki M, Fujii N, Yajima H, Ishii T, Nishizawa Y, Miyazaki K, Katayama T (1995) Biochem 34: 1145311459.

Grant MB, Caballero S, Bush DM, Spoerri PE (1998) Diabetes 47: 1335-1340.

Hashino K, Uemori Y, Kimizuka F, Kato I, Titani K (1996) J Biochem 119: 604-609.

Huynh QN, Wang S, Tafolla E, Gansky SA, Kapila S, Armitage GC, Kapila YL (2002) Periodontal 73: 1101-1110.

James K, Skibinski G (1995) In: Immunology of Human Reproduction. Kurpisz M, Fernandez N, eds, pp 267-283. BIOS Scientific Publishers Ltd.

Labat-Robert J (2002) Cancer Biol 12: 187-195.

Laemmli UK (1970) Nature 227: 680-685.

Lilja H, Oldbring J, Rannevik G, Laurell CB (1987) Am Soc Clin Invest 80: 281-285.

Pinke LA, Swanlund AJ, Hensleigh HC, McCarthy JB, Roberts K, Pryor JL (1997) J Urol 158: 936-941.

Romberger DJ (1997) Int J Biochem Cell Biol 29: 939-943.

Stanton KJ, Frewin MB, Gudewicz PW (1999) J Leukocyte Biol 65: 515-522.

Stauffer ChW, Parsons CL (1989) Urology 34: 80-85.

Tchougounova E, Forsberg E, Angelborg G, Kjellen L, Pejler G (2001) J Biol Chem 276: 3772-3777.

Wassarman P, Litscher ES (2001) Cells Tissues Organs 168: $36-45$.

Wennemuth G, Meinhardt A, Mallidis C, Albrecht M, Krause W, Renneberg H, Aumüller G (2000) Andrology 33: 43-46.

Yamada KM (2000) J Clin Invest 105: 1507-1550.

Yi M, Ruoslahti E (2001) Proc Natl Acad Sci USA 98: 620624. 